\title{
Les amphores Günsenin IV à Küçükyalı (Istanbul). Un voyage entre monastères?
}

\section{Nergis Günsenin et Alessandra Ricci}

\section{(2) OpenEdition}

1 Journals

Édition électronique

URL : https://journals.openedition.org/anatoliaantiqua/598

DOI : 10.4000/anatoliaantiqua.598

Éditeur

IFEA

Édition imprimée

Date de publication : 1 décembre 2018

Pagination : 125-140

ISBN : 9782362450747

ISSN : 1018-1946

\section{Référence électronique}

Nergis Günsenin et Alessandra Ricci, « Les amphores Günsenin IV à Küçükyalı (Istanbul). Un voyage entre monastères ? », Anatolia Antiqua [En ligne], XXVI | 2018, mis en ligne le 30 juillet 2019, consulté le 03 septembre 2021. URL : http://journals.openedition.org/anatoliaantiqua/598 ; DOI : https://doi.org/ 10.4000/anatoliaantiqua.598 


\section{ANATOLIA ANTIQUA ESKI ANADOLU}

\section{XXVI}

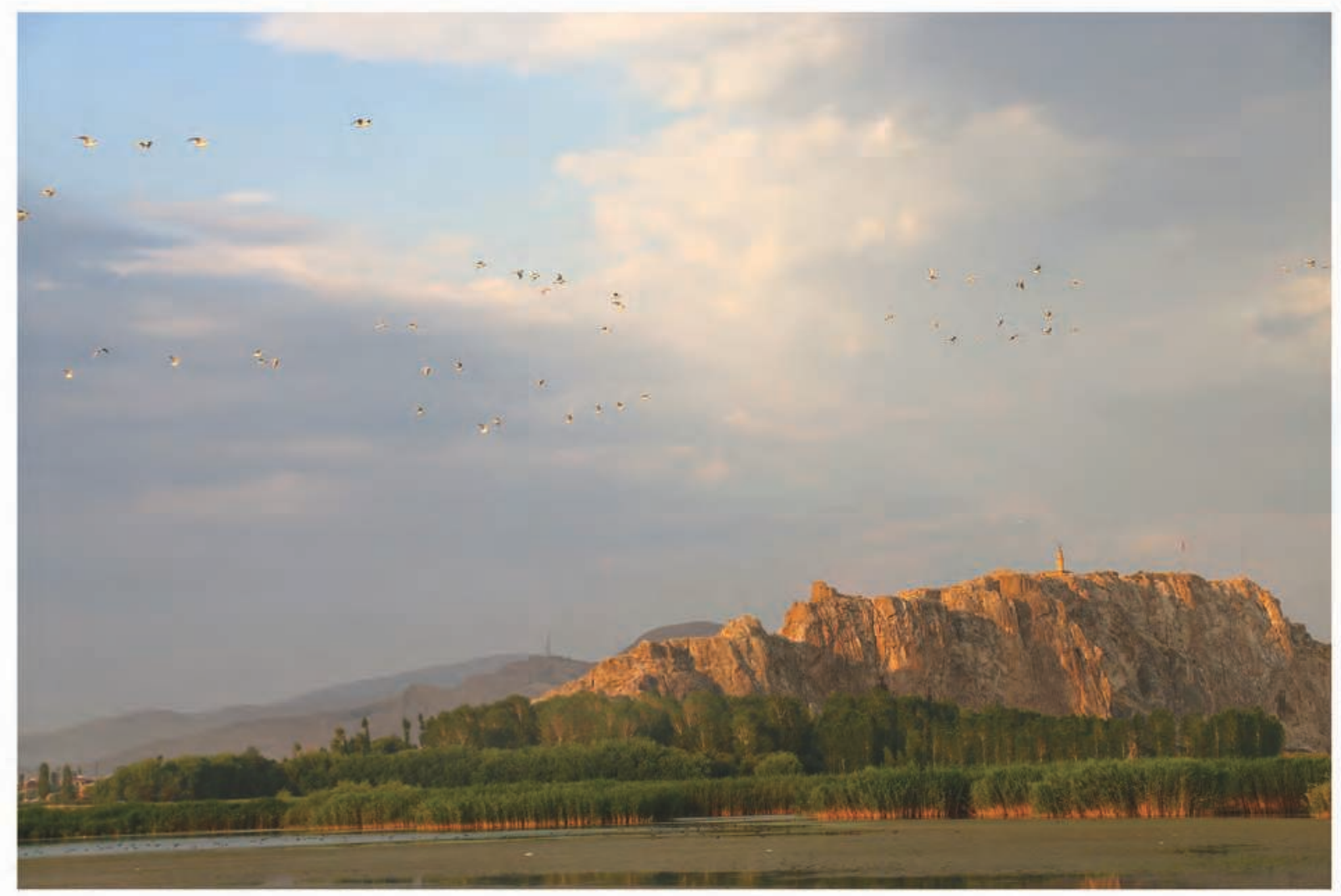

INSTITUT FRANÇAIS D'ETUDES ANATOLIENNES GEORGES-DUMEZIL

CNRS USR 3131

DE BOCCARD 


\section{TABLE DES MATIERES}

Alice VINET et Denis GUILBEAU

A First Glimpse of the Late Neolithic and Early Chalcolithic in Cappadocia through the Lithic

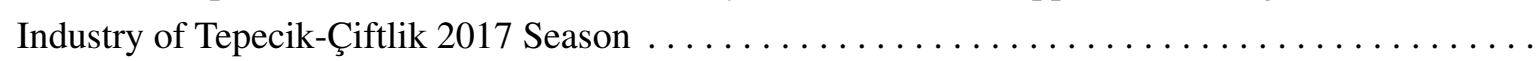

Ergül KODAŞ, Haluk SAĞLAMTIMUR et Yılmaz Selim ERDAL

Three Human Graves of the Hassuna Culture in Türbe Höyük

\section{Kevin PARACHAUD}

Les Galates en Asie Mineure au regard de la culture matérielle. Hellénisation, maintien, acculturation ? 23

H. Asena KIZILARSLANOĞLU et Erkan ALKAÇ

Hellenistic Amphora Stamps from Elaiussa . . . . . . . . . . . . . . . . . . . . .

Aygün EKİN MERİÇ

Late Roman Pottery from the Theatre of Nicaea in Bithynia $\ldots \ldots \ldots \ldots \ldots \ldots \ldots \ldots \ldots$

Nergis GÜNSENIN

La typologie des amphores Günsenin. Une mise au point nouvelle.

Nergis GÜNSENIN et Alessandra RICCI

Les amphores Günsenin IV à Küçükyalı (Istanbul). Un voyage entre monastères ? . . . . . . . . .

\section{CHRONIQUES DES TRAVAUX ARCHEOLOGIQUES EN TURQUIE 2017}

Erkan KONYAR, Bülent GENÇ, H. Banu KONYAR, Armağan TAN et Can AVCI

Excavations at the Old City, Fortress, and Mound of Van: Work in 2017 . . . . . . . . . . . . . .

\section{Çiğdem MANER}

Preliminary Report on the Fifth Season of the Konya Ereğli, Karapınar, Halkapınar and

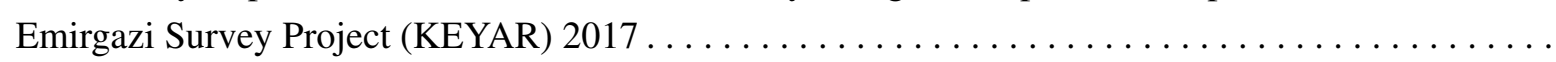

Abuzer KIZIL, Koray KONUK, Taylan DOĞAN, Didier LAROCHE, Enora LE QUERE,

Vasilica LUNGU, Francis PROST et Baptiste VERGNAUD

Eurômos : Rapport préliminaire sur les travaux réalisés en 2017

Olivier HENRY et E. ANDERSSON, J. BLID, Chr. BOST, Ö. ÇAKMAKLI, N. CARLESS-UNWIN, G. ÇİMEN, A. EYİGÖR, A. FRECCERO, A. FREJMAN, Cr. GEORGESCU, E. GOUSSARD, A.-M. GUIMIER-SORBETS, M. HAUCHART, R. HEDLUNG, N. LAMARE, V. LUNGU, Fr. MARCHAND-BEAULIEU, A. SITZ, I. STOJANOVIC, B. VERGNAUD 


\section{LES AMPHORES GÜNSENIN IV A KÜÇÜKYALI (ISTANBUL) UN VOYAGE ENTRE MONASTERES ?}

Quand ma collègue Alessandra Ricci m'a appelée avec enthousiasme pour m'informer de la découverte de deux amphores du type Günsenin IV lors de leur campagne de fouilles en octobre 2015, je me suis immédiatement retrouvée sur le site de Küçükyalı. Suite à une enquête rapide, ma surprise fut grande de constater qu'une estampille attestée sur les amphores de notre épave de Çamaltı Burnu I pouvait être restituée sur les deux anses de l'une d'entre elles (voir infra Fig. 4). Dans les deux cas on trouve les deux lettres grecques kappa et oméga avec ligature. Une légère différence stylistique peut être constatée dans le motif en forme d'amande qui entoure le cercle, peut-être un omicron, sur l'exemplaire de Çamaltı Burnu I (voir infra Fig. 5). La lecture la plus probable

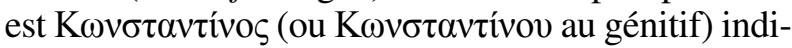
quant sans doute le nom du producteur du contenu de l'amphore. Une autre lecture, moins vraisemblable, pourrait donner K $\omega \nu \sigma \tau \alpha \nu \tau \imath v o v ́ \pi \circ \lambda \eta^{1}$.

Le travail sur les amphores de Küçükyalı se poursuit et il y a de fortes chances que d'autres amphores portant la même estampille puissent être identifiées. La récurrence de la même estampille à Küçükyalı et dans l'épave de Çamaltı Burnu I vient conforter l'hypothèse du lien que j'ai proposé de dresser entre l'épave et ce monastère ${ }^{2}$.

J'admets avec regret que j'avais négligé jusquelà de rendre visite à la fouille de Küçükyalı ArkeoPark (parc archéologique-KYAP), menée par les Musées archéologiques d'Istanbul sous la direction scientifique d'Alessandra Ricci depuis 2014.

Ricci décrit le site comme le monastère de Satyros édifié par le patriarche Ignace dans les années
867-877. La chronologie des petits objets découverts sur le site, fournissant des dates allant du $5^{\mathrm{e}}$ au $7^{\mathrm{e}}$ siècle, montrent une vie préalable au monastère. Deux monnaies des règnes d'Andronic II (1282-1328) et d'Andronic III (1328-1341) montrent que l'établissement était actif jusqu'au debut du $14^{\mathrm{e}}$ siècle ${ }^{3}$.

L'intérêt que ce site a éveillé en moi n'a pas été uniquement motivé par son importance historique et l'étude des amphores. En effet, j'ai également été très impressionnée par le projet du parc archéologique (ArkeoPark) tel qu'il a été adapté à la vie de la communauté voisine et particulièrement, l'enseignement de notre patrimoine culturel aux enfants ${ }^{4}$. J'ai donc intégré l'équipe avec enthousiasme et j'ai commencé à étudier les matériaux. (N.G.)

\section{THE SITE AT KÜÇÜKYALI (A.R.)}

Tucked in between modern apartment buildings and set slightly inland from the Marmara seashore, the archaeological site at Küçükyalı is located at a close proximity to the Prinkipo islands (modern Adalar), and extends over some 4,500/5,000 sq. meters. The area was part of the capital city's extended Asian hinterland in Byzantine times.

The surviving remains at the site center around a rectangular-in-plan earth platform $(62 \times 50 \mathrm{~m}$, with a $15.2 \mathrm{~m}$ continuation of it detected to the north-west) that is supported by retaining walls. The lower level of the platform, which was completely underground in antiquity, was occupied in part by an east-west oriented cistern with a water feeding channel to the east of the structure. The larger portion of the cistern was originally covered by

*) Prof. Dr. Nergis GÜNSENIN, İstanbul Üniversitesi-Cerrahpaşa,Teknik Bilimler Meslek Yüksekokulu, Motorlu Araçlar ve Ulaştırma Teknolojileri Bölümü, Sualtı Teknolojisi Program Başkanı, Büyükçekmece Yerleşkesi, 34500-Istanbul, e- mail : gunsenin@istanbul.edu.tr

Doç. Dr. Alessandra RICCI, Department of Art and Archaeology, Koç University, Rumeli Feneri Yolu, 34450 Sarıyer- İstanbul. E-mail: aricci@ku.edu.tr

1) Je remercie Frederick H. van Doorninck, Jr. pour la lecture et des commentaires sur le monogramme.

2) Pour l'épave de Çamaltı Burnu I, voir notamment, Günsenin 2001 ; 2003 ; 2005 ; Günsenin (sous-presse). Cf., aussi, la bibliographie complète de N. Günsenin dans Günsenin 2018 dans ce volume pp. 89-124.

3) Ricci $2012: 147-162$.

4) Ricci et Y1lmaz $2016: 41-62$. 
28 brick domes resting either on piers or columns arranged in four parallel rows. The original roofing system of the smaller eastern portion of the cistern is preserved, and is dominated by a monumental brick dome at the center, which rests on four massive stone-and-brick piers. Lateral corridors defined by barrel vaults and groin vaults at the corners frame the dome, creating a square. The cistern could hold some 2,700 cubic meters of water, although sinter deposits on its waterproof reveted walls indicate that it was not continually used in its full capacity. The cistern was underground at the time of its construction with a massive earth fill that formed the rectangular-in-plan platform above $i^{5}$.

Archaeological investigations verified the presence of ramps leading to the elevated platform. Wall remains above the platform, more specifically above the eastern portion of the cistern, were visible until the mid-1990s. These were identified as the remains of an ecclesiastical building, already noted by Pargoire and Mamboury at the beginning of the $20^{\text {th }}$ century, and later as an Islamicate palace ${ }^{6}$. In particular, Mamboury recorded the presence of Byzantine-period brick stamps at the site and identified the remains above the cistern as the church of the monastery of Satyros, built by the patriarch eunuch Ignatios during his tenure at the seat of Constantinople ${ }^{7}$.

The subsequent study by Eyice, which included the first surveyed plan of the remains visible at the time, focused on the cistern and its hypothesized building phases, and concluded that the complex had the architectural characteristics of an Islamicate palace $^{8}$. These were palaces built in the Umayyad and Abbasid times in the desert and semi-desert regions of the caliphates such as Mshatta (in modern Jordan) and Ukhaidir (in modern Iraq). On their return to Constantinople from Baghdad, Byzantine-period envoys persuaded the emperor Theophilos to build a similar palace 9 .

\section{ARCHAEOLOGICAL INVESTIGATIONS AND THE INTERPRETATION OF THE SITE (A.R.)}

Fieldwork followed by archaeological excavations helped clarify the issues concerning the iden- tification of the complex by bringing into question the presence of an Islamicate palace at Küçükyal ${ }^{10}$.

The following is a concise summary of the main architectural and archaeological data gathered on the site that do not support the identification of the remains as those of a palatine complex (Fig. 1).

Analysis of the cistern, believed to represent the core of the Islamicate palace in its primary building phase, revealed that this lower level underground structure was meant to serve as a water reservoir from the very beginning. The opening within its western perimeter wall that Eyice labeled as the entrance into the "lower level space" is the result of a modern piercing of the wall to provide access into the by then abandoned and no longer functioning cistern. Furthermore, a 3D laser scan survey of the cistern conducted in 2015 and 2016 indicated that the cistern and the remains above it were structurally bonded, confirming that the complex was built concurrently ${ }^{11}$.

On the platform and above the eastern portion of the cistern that is dominated at the underground level by a brick dome resting on four massive piers, conspicuous remains of the ecclesiastical building observed by Mamboury were detected. The church on the platform and the cistern underneath were physically connected, as the 3D laser scan indicated. Furthermore, as excavation of the church begun in 2014, it was possible to confirm that the building was equipped with a central dome resting on four piers. The piers are axially aligned with the piers underneath, although they are smaller in size and present a more elaborate shape.

The building's rather emphatic lateral entrances, with the one on the north excavated in 2015 and 2016, are aligned with the access ramps to the platform, constituting a further indication of the cohesiveness of the architectural plan and of its execution. The ecclesiastical building is preserved to a maximum height of $1 / 1.20 \mathrm{~m}$, showing a solid brick construction technique comparable to the Myrelaion church (modern Bodrum camii) ${ }^{12}$.

As for the plan of the building, it is now possible to calculate the length of the east-west axis of the church as approximately $21 \mathrm{~m}$ after discovery of unsubstantial remains of its narthex in the north-

5) Ricci $1998: 131-149$.

6) For the ecclesiastical building, see Pargoire $1901: 62-78$; Mamboury $1920: 322-330$. For the Islamicate palace, see Eyice $1959: 79-114$.

7) Mamboury $1920: 328-330$. For the brickstamps recorded by Mamboury, see Bardill 2004, Vol. I : 418.

8) Eyice 1959 : pl. 2.

9) Eyice $1959: 95-100,101-104$

10) Ricci $1998: 131-149$.

11) Ricci $2017: 141-143$, fig. 3, 138.

12) Ricci $2017: 141-143$ 


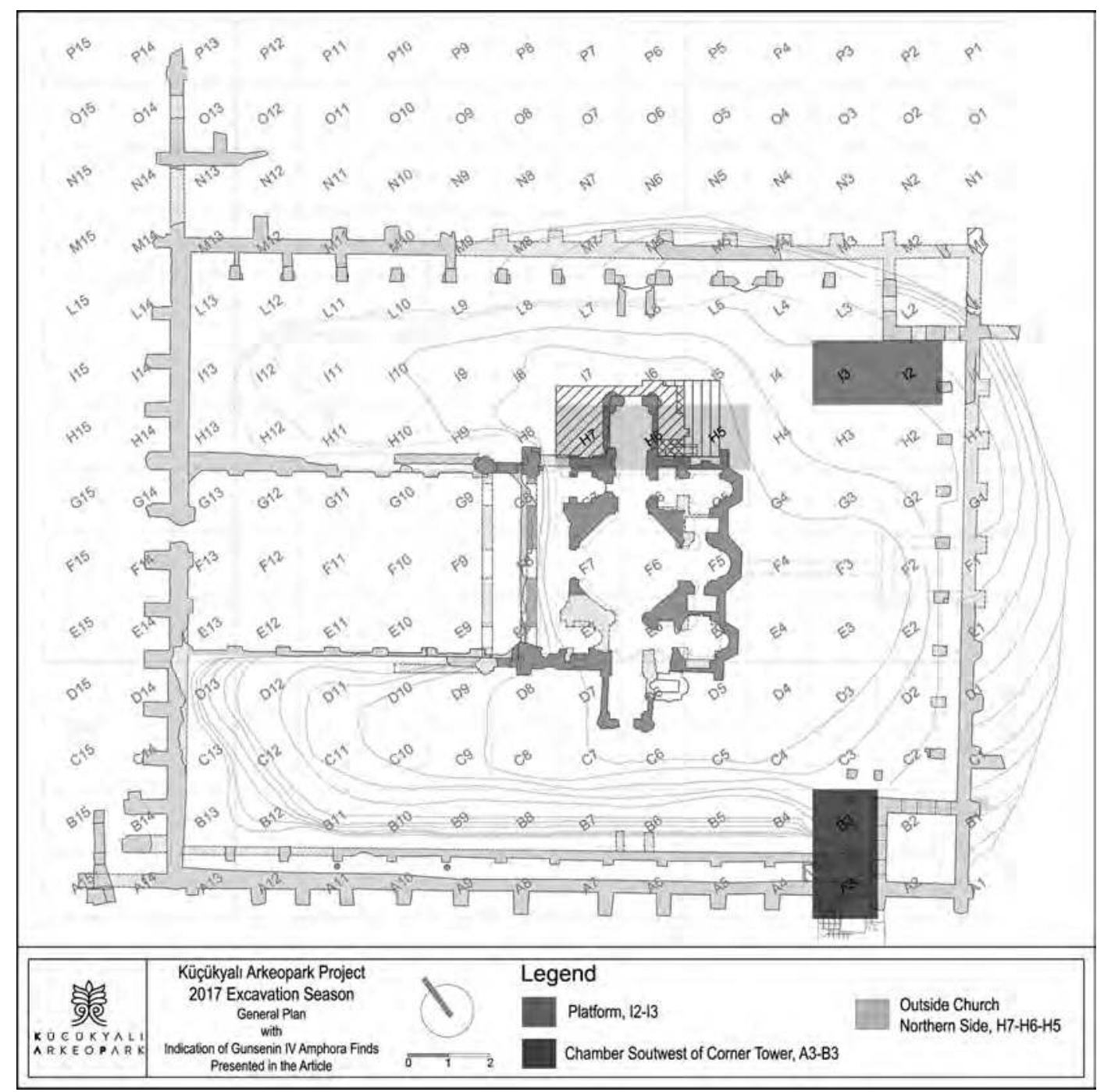

Fig. 1 : Plan général du site de Küçükyalı (KYAP Archive).

western area. The possibility of the presence of an atrium to the west of the narthex, resting above the cistern's western portion where its brick domes have collapsed, should not be excluded. Based on survey and excavation data, the width of the church is currently calculated as $18 \mathrm{~m}(21 \mathrm{x} 18 \mathrm{~m}$ or $21 \mathrm{x} 24 \mathrm{~m}$ where the lateral entrances are). The ecclesiastical building appears to be of rather large dimensions for the middle Byzantine period. It is likely to be representative of cross-domed churches with a tripartite apse, emphatic lateral entrances, side chapels, and a narthex that may have connected to an atrium.

The architectural sculpture decoration of the building retrieved during excavations consists of wall cornices, parapet slabs, iconostasis fragments, as well as window elements. It clearly represents the creative expression of a workshop with distinc- tive Constantinopolitan features. The material from the early $10^{\text {th }} \mathrm{c}$. northern church of the monastery of Constantine Lips in Constantinople represents a good example for comparison ${ }^{13}$. The church was also decorated with wall mosaics that survived in very fragmentary condition. Fragments of human eyes and of human garments stand out among them for the purpose of identification of the complex, offering further confirmation that the mosaics must not have been produced during the iconoclastic period.

The identification of the complex with the monastery of Satyros or Anatellos (the Rising One) is further reinforced by the discovery of a funerary chapel hastily added to the exterior of the southeastern wall of the church. Its location appears to fit with the description of the burial of patriarch Ignatios (d. October 23, 877) in the vita composed by 
David Niketas Paphlago ${ }^{14}$. It is therefore confirmed that the complex at Küçükyalı can be identified with the monastery built by Ignatios on the Marmara seashore across from the Princes islands, where he erected two more monasteries.

Work on the platform of the complex included the excavation of its northeastern and southeastern corners, where electromagnetic surveying showed the presence of architectural features. In particular, the northeastern corner of the platform yielded a Günsenin IV amphora in 2015; a second one was assembled in 2016 after excavation of the chamber to the southwest of the platform's southeastern tower, and a third one was assembled also in 2016 after the excavation of the area adjacent to the katholikon's external northern wall and lateral entrance. These finds represent the focus of the study by Nergis Günsenin.

\section{LA SAISON DE LA FOUILLE DE 2015 (N.G.)}

\section{Type : Günsenin IV ${ }^{15}$}

\author{
Numéro d'inventaire \\ : KY/15 ; US 1504 (Fig. 2) ${ }^{16}$. \\ Lieu de conservation \\ : Musées archéologiques \\ d'Istanbul. \\ Lieu de découverte \\ : Dans la plate-forme à la \\ section nord-est du \\ complexe. Carrée I3 \\ (platform area) (Fig. 3) ${ }^{17}$. \\ Etat de conservation \\ : Les deux tiers ont été \\ complétés. \\ Dimensions \\ : hauteur : 73,7 cm. ; avec les \\ anses : $80 \mathrm{~cm}$. \\ : diamètre embouchure : \\ $8,6 \mathrm{~cm}$. \\ : diamètre panse : $67,2 \mathrm{~cm}$. \\ Pâte \\ : Surface extérieure : \\ self-slip. \\ : Intérieur de la pâte : \\ pâte fine, orange soutenu.
}

Capacité approximative

\author{
Inscription \\ Parallèles \\ Commentaire
}
: Estampage sur les deux anses avant cuisson (Fig. 4).
: Cargaison de l'épave de Çamaltı Burnu I (Fig. 5) ${ }^{19}$.
: Des traces (entailles) sur la surface interne de la panse proviennent de la phase de production. Ces marques ont été faites pendant le contrôle du diamètre interne de la panse. C'est un procédé commun aux jarres, amphores Günsenin IV présentant de grands volumes (Fig. 6) ${ }^{20}$.

\section{Type : Günsenin IV ${ }^{21}$}
Numéro d'inventaire $\quad$ : KY/10 ; US 1073 (Fig. 7) 22 .
Lieu de conservation : Musées archéologiques d'Istanbul.
Lieu de découverte : Carrée A3 (chamber) ${ }^{23}$.
Etat de conservation : Les deux tiers ont été complétés.
Dimensions : Hauteur : $52,4 \mathrm{~cm}$; avec les anses : $57 \mathrm{~cm}$.
: diamètre embouchure : $7 \mathrm{~cm}$.
: diamètre panse : 45
: self-slip
: Intérieur de la pâte : pâte fine, orange soutenu.
Capacité approximative : 42,6 litres ${ }^{24}$
Parallèles : L'épave de Çamaltı Burnu I.

Commentaire : Des traces (entailles) sur la surface interne de la panse proviennent de la phase de production. Ces marques ont été faites pendant le contrôle du diamètre interne de la panse. Il s'agit d'un procédé commun aux jarres, amphores

14) Smithies $2013: 108-109$.

15) Le type/classe Günsenin IV a été divisé en six sous types par l'auteur dans sa thèse de doctorat. Voir la discussion à propos du type/classe dans Günsenin 1990 : 31-34 et Günsenin 2018, dans ce volume pp. 89-124. Les recherches en cours nous apporteront des éléments neufs sur la manière de classifier les amphores de l'épave de Çamaltı Burnu I en raison du fait qu'elles montrent une forme différente parmi les six sous types!

16) Cf. aussi, dans Günsenin (sous-presse).

17) Les fouilleurs décrivent qu'elle a été trouvée entière et a pu avoir été brisée par le poids du sol au-dessus.

18) Voir Addendum.

19) Les monogrammes de l'épave sont en cours d'étude pour la publication finale.

20) Cf. Volkov $1992: 146$ et 147, Fig. 2.

21) Voir supra note 15.

22) Cf. Ricci et Wohmann 2018, Vol I : 456, Fig. 3.25

23) Voir dans Fig. 1

24) Voir Addendum. 

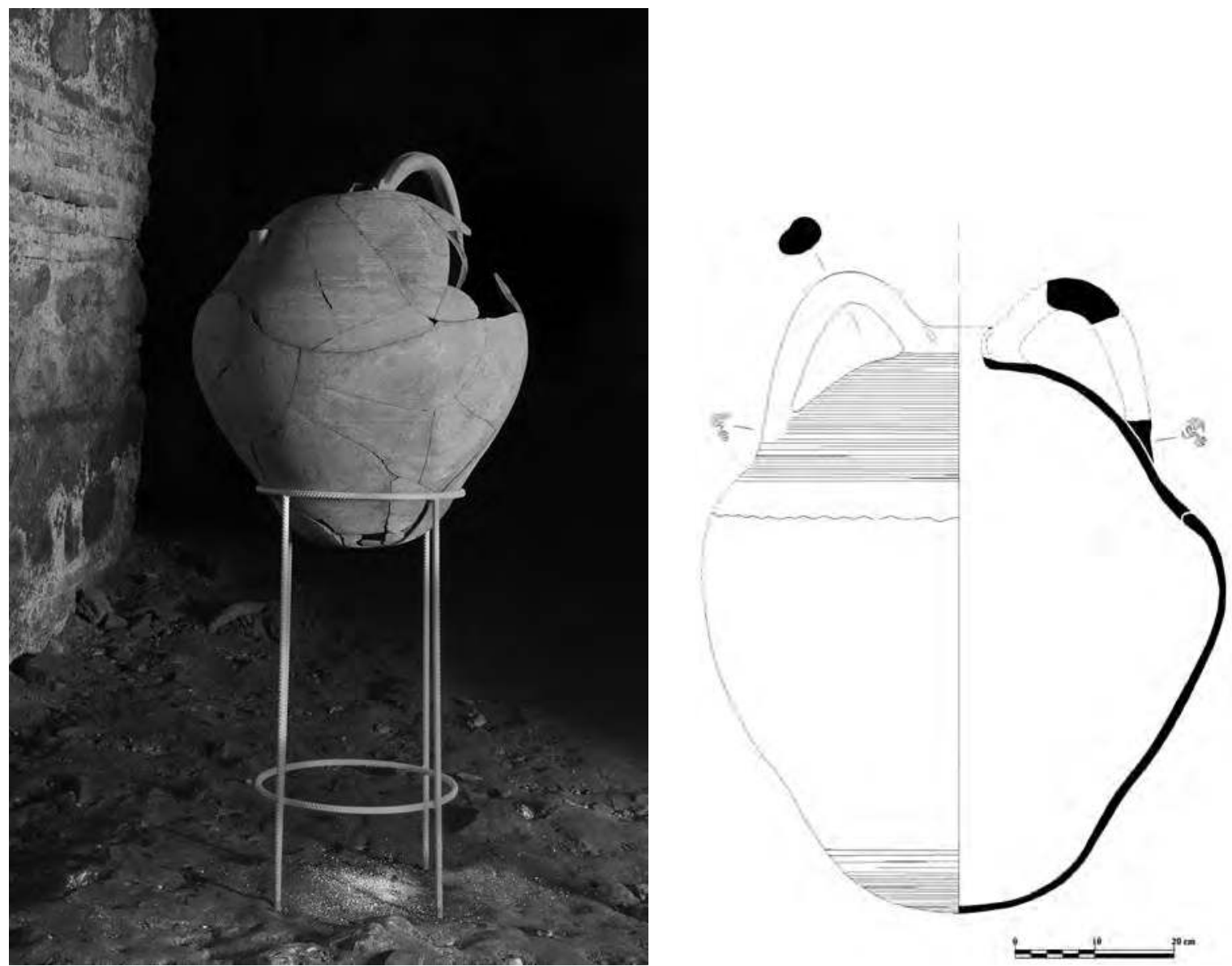

Fig 2 : Amphore Günsenin IV : a-b. KY/15 ; US 1504 (Photo: Domenico Ventura. KYAP Archive. Restoration et dessin de l'amphore : Taner Özgür).

Günsenin IV qui présentent de grands volumes (Fig. 8) ${ }^{25}$. On observe un trou de fausset, fait avant cuisson sur l'un des fragments de la panse ${ }^{26}(\text { Fig. 9 })^{27}$.

\section{LA SAISON DE LA FOUILLE DE 2016 (N.G.)}

\section{Type : Günsenin IVf ${ }^{28}$}

Numéro d'inventaire

: KY/16 ; US 2612, 2627

(Fig. 10).

Lieu de conservation : Musées archéologiques d'Istanbul.

Lieu de découverte

: Les fragments restitués venant des carrées H5, H6, H7 (church area).
Etat de conservation

Dimensions

Pâte

Capacité approximative Graffiti
: Les deux-tiers ont été complétés.

: hauteur conservée

: $48,8 \mathrm{~cm}$; avec les anses

: $53 \mathrm{~cm}$.

: diamètre embouchure : $6,2 \mathrm{~cm}$.

: diamètre panse : $50 \mathrm{~cm}$.

: Surface extérieure : self-slip

: Intérieur de la pâte

: pâte fine, orange claire.

: 45 litres $^{29}$.

: En haut de la panse. A deux endroits de la surface de la jarre.

25) Voir supra note 20.

26) C'est un fragment isolé appartenant à notre amphore. Nous attendons la poursuite de la fouille pour tenter sa restauration.

27) L'apparition de trous (trous de fausset, faits normalement après la cuisson) nous indique une probable fermentation de vin dans l'amphore. Sur ce sujet, voir Adan-Bayewitz 1986.

28) Cf. Günsenin $1990: 32$ et Günsenin 2018, dans ce volume pp. 89-124.

29) Voir Addendum. 


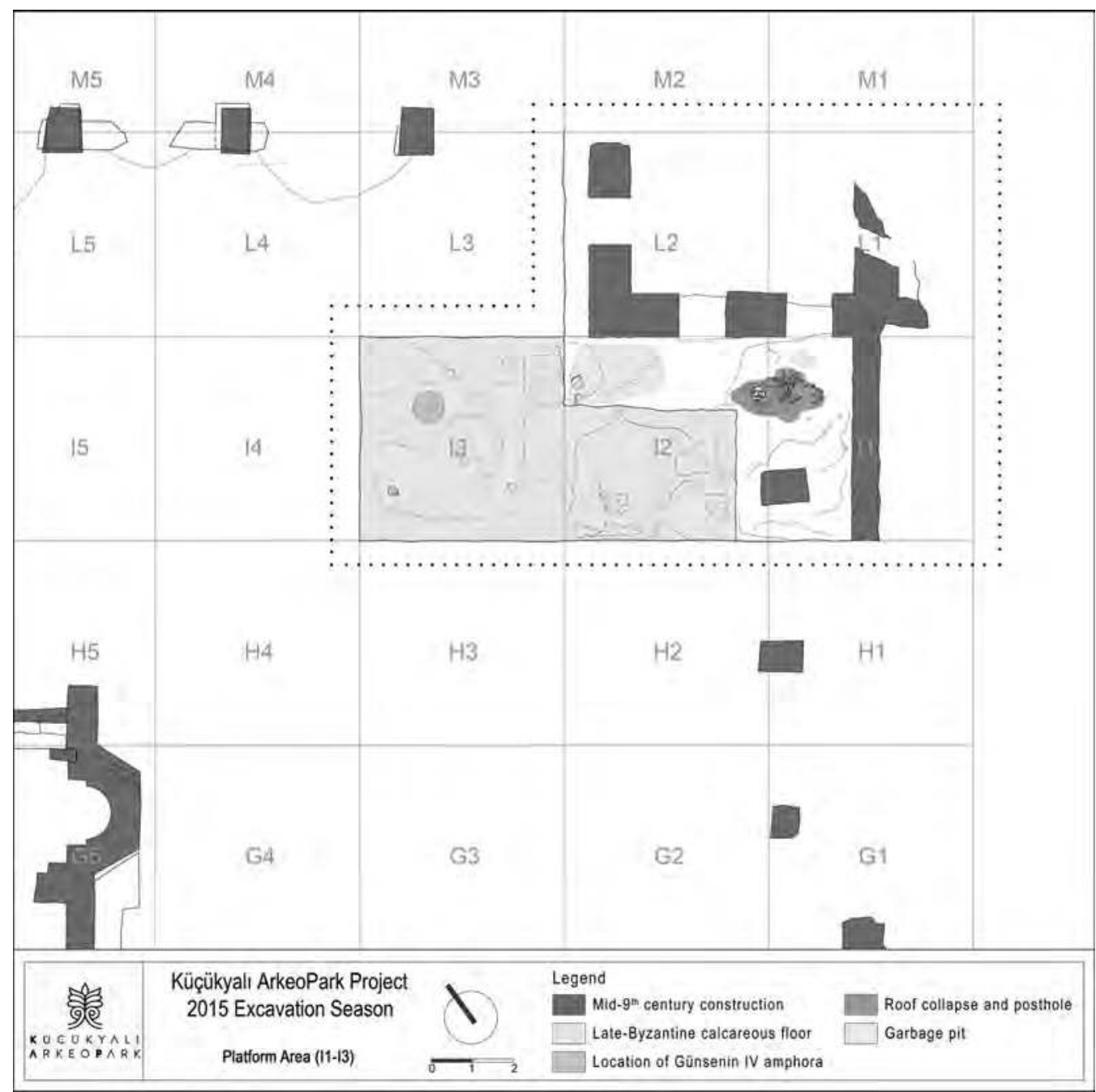

$\mathbf{a}$

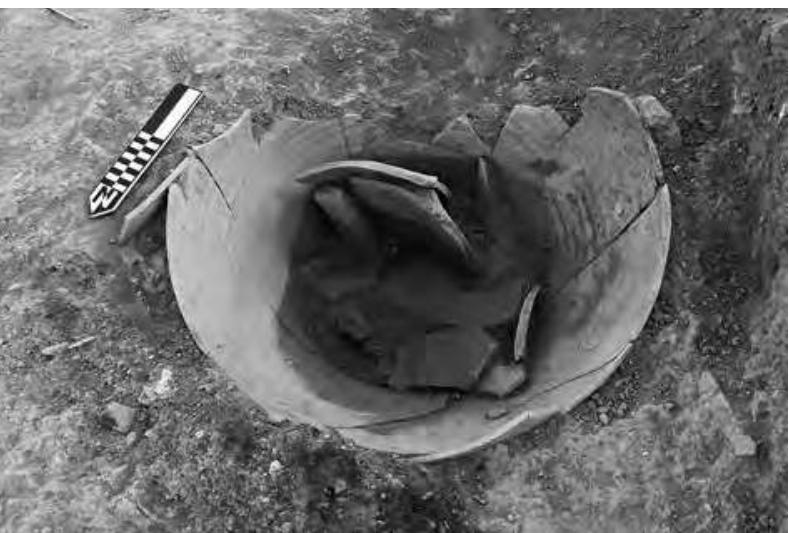

b

Fig. 3 : a. Plan du site avec l'emplacement de l'amphore Günsenin IV, KY/15 ; US 1504 (KYAP Archive) ; b. L'emplacement in situ de l'amphore Günsenin IV, KY/15 ; US 1504 (KYAP Archive).
Commentaire : Un trou de fausset d'un diamètre de $0,9 \mathrm{~cm}$ fait avant cuisson s'observe en haut de la panse (Fig. 11) ${ }^{30}$. Traces de résine à l'intérieur de la jarre. Traces de brûlure/cendres sur la panse.

Les fouilles sont actuellement en cours. Il serait préférable d'attendre l'achèvement des fouilles des tranchées. Nous avons néanmoins décidé de restituer les fragments recueillis sans attendre le reste qui, sûrement, sera mis au jour dans les saisons à venir. Notre décision de restauration a eu un bon résultat. Elle nous a donné une amphore avec une panse conique, très large à mi-hauteur avec une haute épaule convexe. Notre amphore est bien différente de celles qui furent trouvées l'année précédente. Une amphore correspondant au type Günsenin IVf, qui a pu être restituée, est le témoignage le plus marquant de l'intérêt de la restauration.

30) Voir supra note 27. 


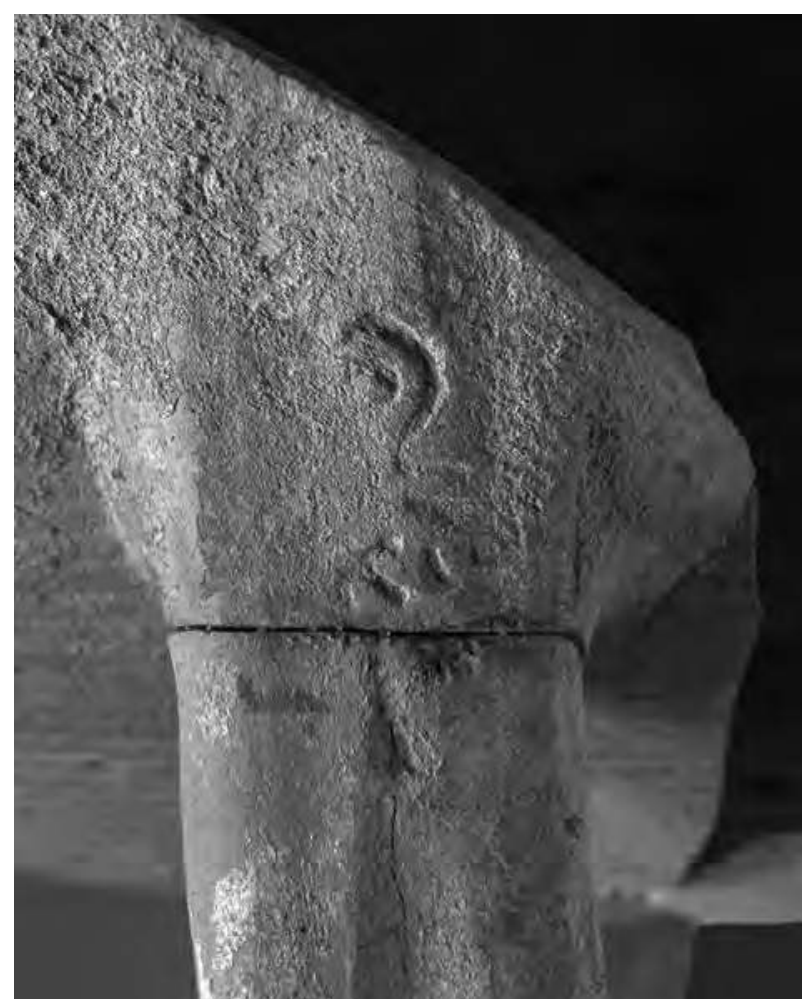

a

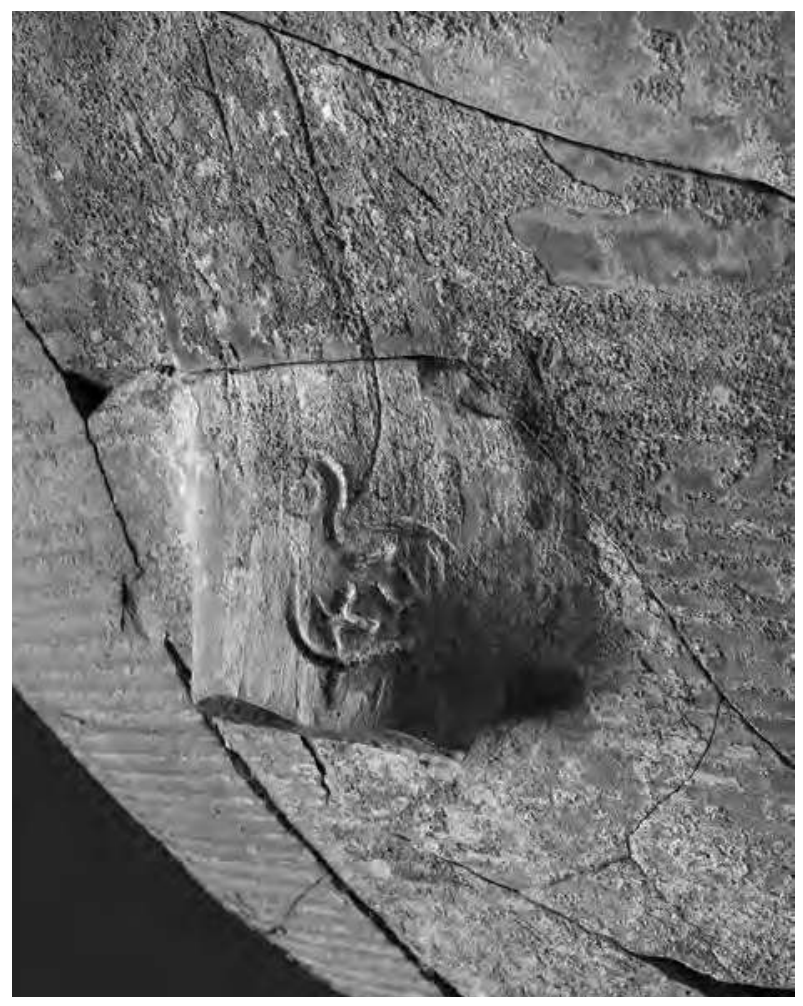

c

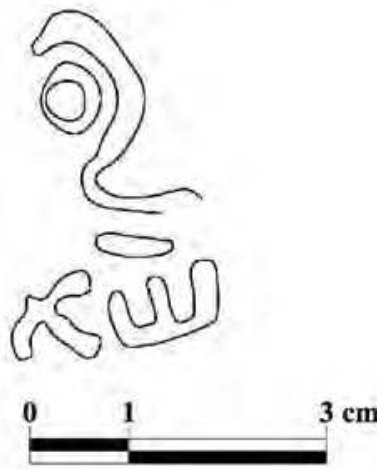

b
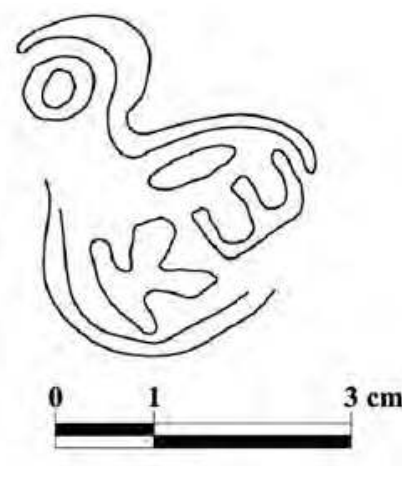

d

Fig. 4 : a-d. Détails des monogrammes sur deux anses de l'amphore Günsenin IV, KY/15 ; US 1504 (Photo : İlksen Baysaling. KYAP Archive. Dessin : Taner Özgür). 


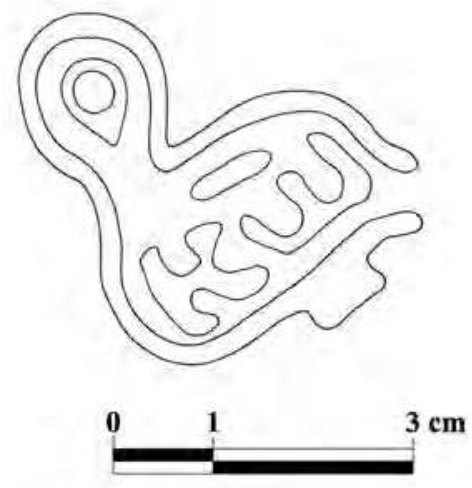

Fig. 5 : Monogramme attesté sur trois amphores de l'épave de Çamaltı Burnu I (Dessin : Taner Özgür).

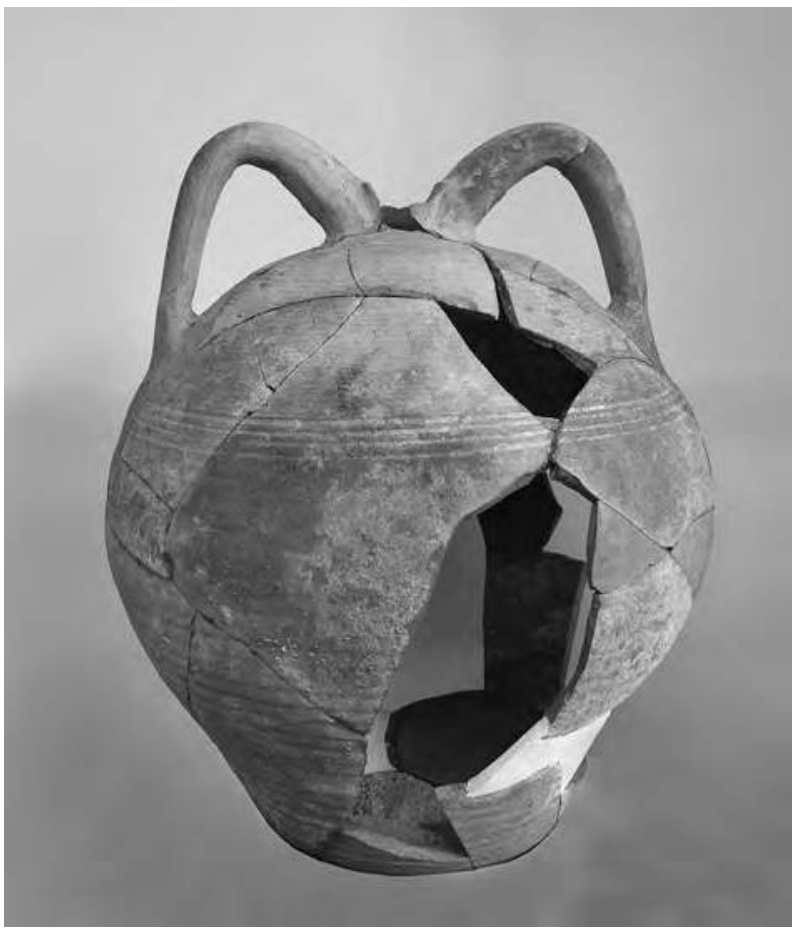

$\mathbf{a}$

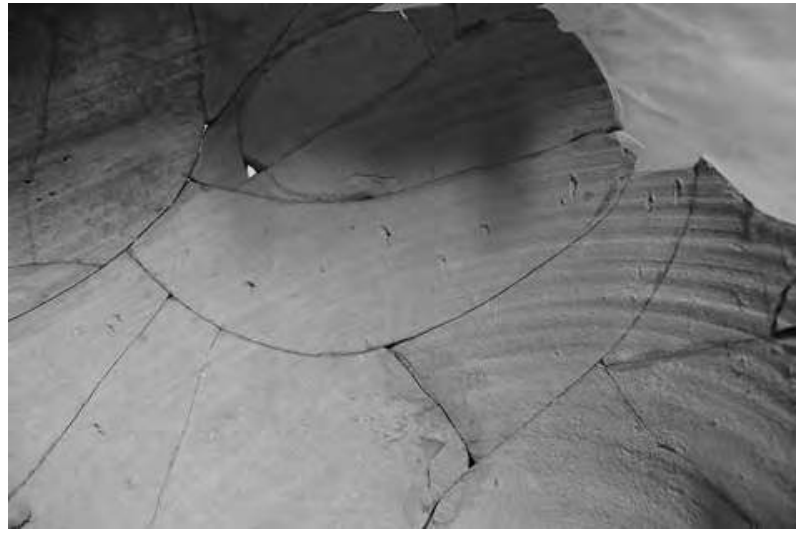

Fig. 6 : Traces (entailles) sur la surface interne de la panse de l'amphore Günsenin IV, KY/15 ; US 1504 (Photo : Taner Özgür. KYAP Archive).

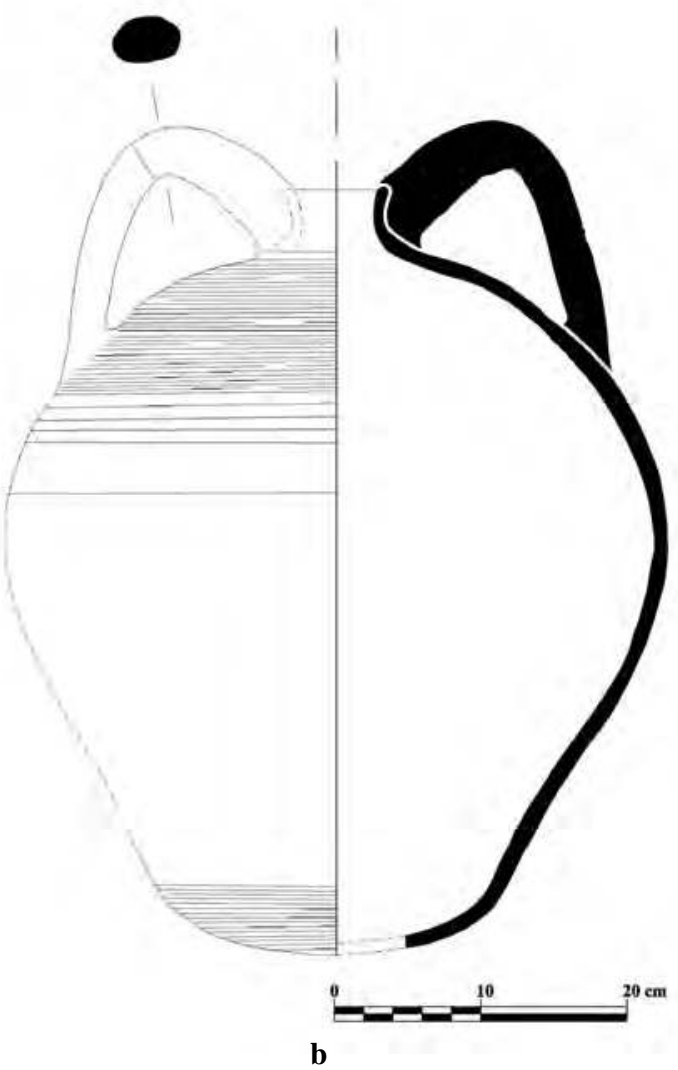

Fig. 7 : a-b. Amphore Günsenin IV, KY/10 ; US 1073

(Photo : Domenico Ventura KYAP Archive. Restoration et dessin de l'amphore : Taner Özgür).

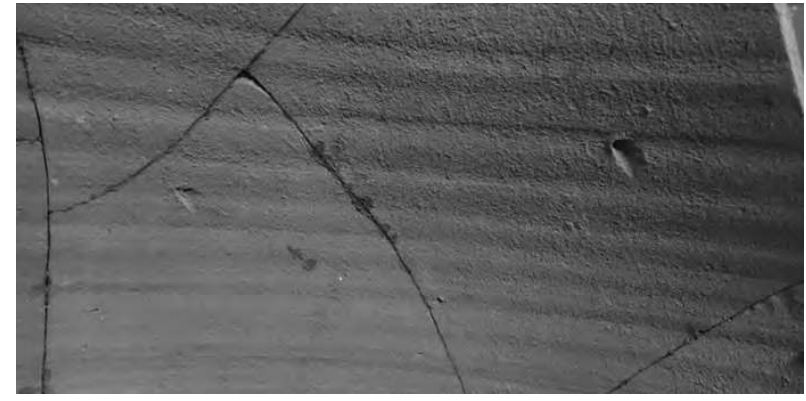

Fig. 8 : Traces (entailles) sur la surface interne de la panse de l'amphore Günsenin IV, KY/10 ; US 1073 (Photo :

Taner Özgür. KYAP Archive). 


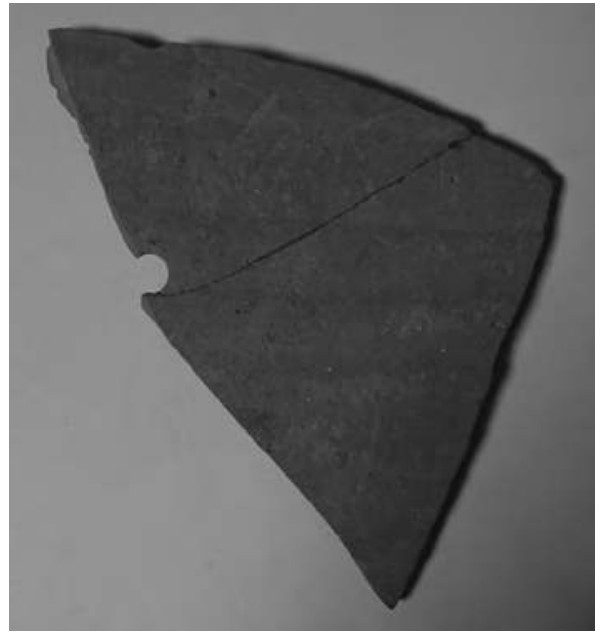

Fig. 9 : Trou de fausset sur un des fragments de la panse de l'amphore Günsenin IV, KY/10 ; US 1073 (Photo : Taner Özgür. KYAP Archive).

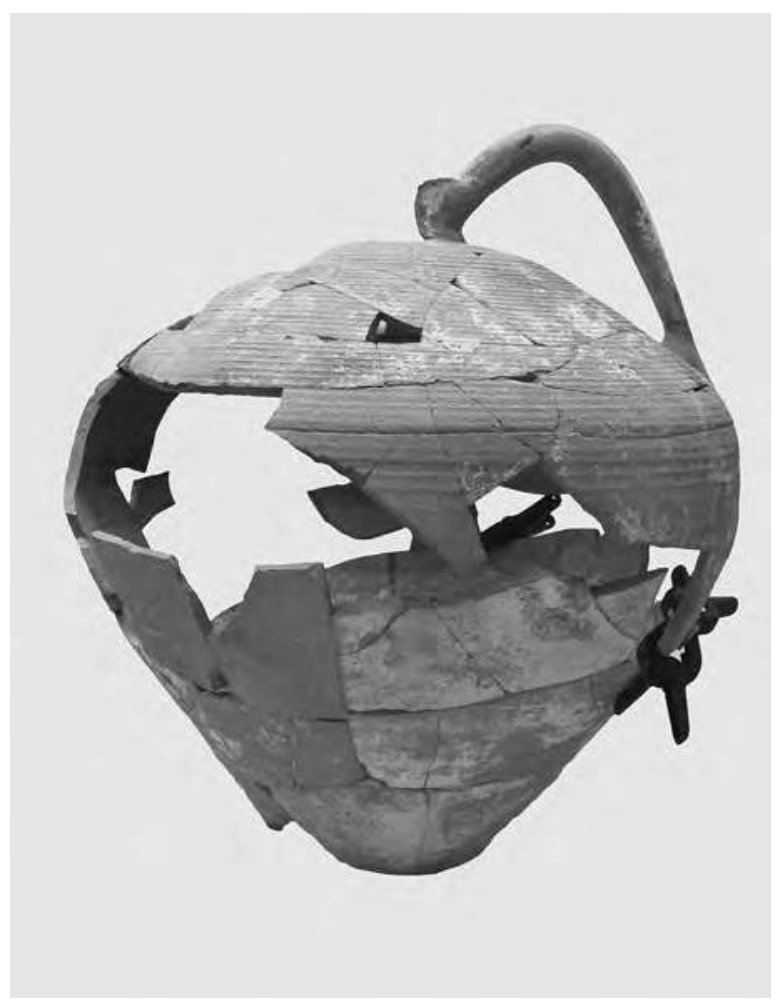

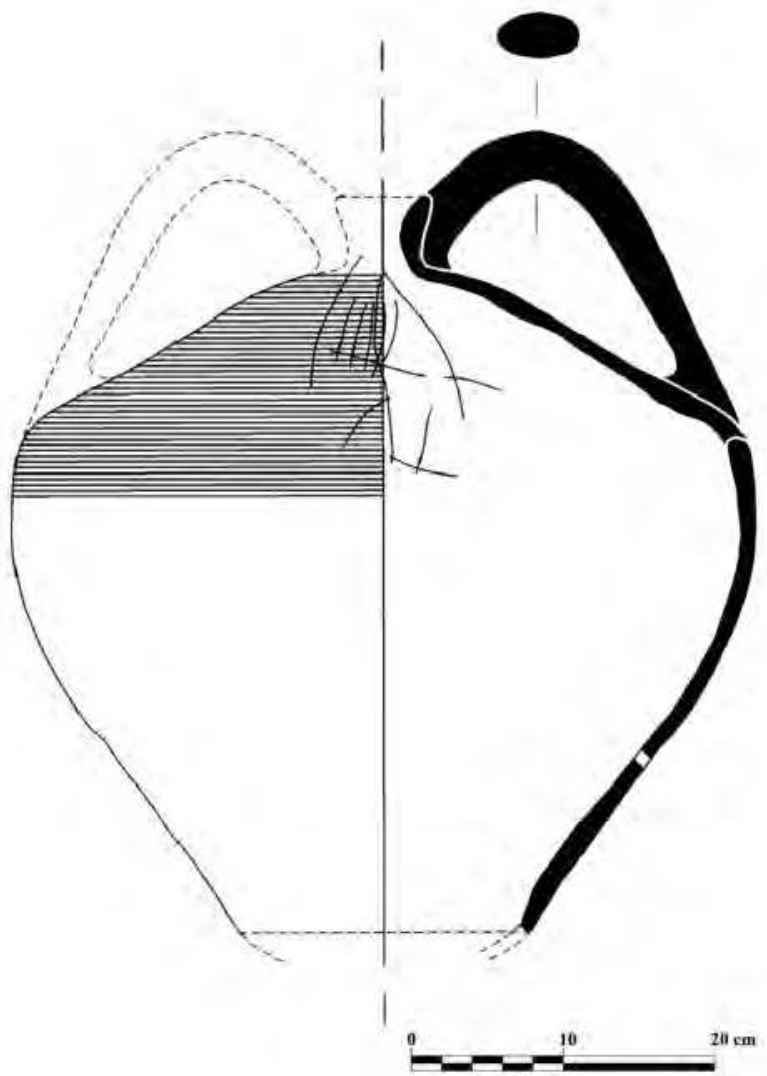

b

Fig. 10 : a-b. Amphore Günsenin IVf, KY/16 ; US 2612, 2627 (Photo : Domenico Ventura. KYAP Archive. Restoration et dessin de l'amphore : Taner Özgür).

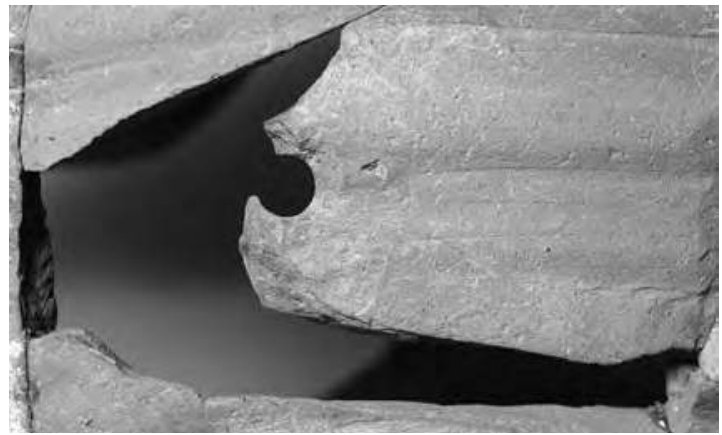

Fig. 11 : Trou de fausset en haut de la panse de l'amphore Günsenin IVf, KY/16 ; US 2612, 2627 (Photo: Domenico Ventura. KYAP Archive). 


\section{Les anses de Günsenin IV}

\begin{tabular}{|c|c|}
\hline Numéro d'inventaire & $\begin{array}{l}\text { KY/16 ; US 2612, } 2627 \\
\text { (Fig. 12). }\end{array}$ \\
\hline Lieu de conservation & $\begin{array}{l}\text { Musées archéologiques } \\
\text { d'Istanbul. }\end{array}$ \\
\hline Lieu de découvert & $\begin{array}{l}\text { : Carrée H5. Secteur de } \\
\text { l'église (Church area). }\end{array}$ \\
\hline Etat de conservation & : Restitution d'une anse. \\
\hline Dimensions & $\begin{array}{l}: 5,1 \text { x } 3 \mathrm{~cm} . \\
: \text { diamètre embouchure : } \\
6,6 \mathrm{~cm} .\end{array}$ \\
\hline Pâte & $\begin{array}{l}\text { Rouge soutenu, grossière } \\
\text { avec du dégraissant. }\end{array}$ \\
\hline Numéro d'inventaire & : KY/16 ; US 2612 (Fig. 13) \\
\hline Lieu de conservation & $\begin{array}{l}\text { : Musées archéologiques } \\
\text { d'Istanbul. }\end{array}$ \\
\hline Lieu de découverte & $\begin{array}{l}\text { : Carrée H6. Secteur de } \\
\text { l'église (Church area). }\end{array}$ \\
\hline Etat de conservation & : Une anse. \\
\hline Dimensions & $: 4 \times 2,4 \mathrm{~cm}$ \\
\hline Pâte & $\begin{array}{l}\text { Beige. Traces de résine et de } \\
\text { cendre à l'extérieur de } \\
\text { l'embouchure. }\end{array}$ \\
\hline Numéro d'inventaire & : KY/16 ; US 2627 (Fig. 14) \\
\hline Lieu de conservation & $\begin{array}{l}\text { : Musées archéologiques } \\
\text { d'Istanbul. }\end{array}$ \\
\hline Lieu de découverte & $\begin{array}{l}\text { : Carrée H6. Secteur de } \\
\text { l'église (Church area). }\end{array}$ \\
\hline Etat de conservation & $\begin{array}{l}\text { : Moitié de l'anse et de } \\
\text { l'embouchure. }\end{array}$ \\
\hline Pâte & : Oxydée. \\
\hline Numéro d'inventaire & : KY/16 ; US 2612 (Fig. 15) \\
\hline Lieu de conservation & $\begin{array}{l}\text { : Musées archéologiques } \\
\text { d'Istanbul. }\end{array}$ \\
\hline Lieu de découvert & $\begin{array}{l}\text { : Carrée H7. Secteur de } \\
\text { l'église (Church area). }\end{array}$ \\
\hline Etat de conservation & : Une anse. \\
\hline Dimensions & : 4,9 x 3,7 cm. \\
\hline Pâte & : Beige orangé. \\
\hline
\end{tabular}

\section{COMMENTAIRES (N.G.)}

La première découverte en 2015 m'avait donné l'idée d'une amphore trouvée de façon isolée, qui a simplement été réutilisée. Mais la deuxième trouvaille ainsi que le montant fragmentaire ont incité une réflexion plus profonde. La saison de fouille de 2016 a aussi été assez riche en matière de découvertes d'amphores Günsenin IV. Au début, j'ai pensé que ces amphores ont été vidées de leur contenu d'origine pour être réutilisées comme cela est souvent le cas pour les amphores byzantines. Mais les bouchons trouvés pendant la fouille (fragments d'amphores arrondies) indiquent que les amphores sont arrivées (du moins certaines) sur place avec leur contenu (Fig. 16). De centaines de fragments de panses ainsi que plusieurs anses, qui permettent de restituer le profil, furent découverts. Les différences des anses ainsi que la forme et la pâte montrent des parentés avec les amphores de l'épave de Çamaltı Burnu I. La recherche menée en cours avec Frederick van Doorninck Jr. montre qu'une partie des amphores Günsenin IV sur le bateau était des jarres réutilisées. L'assemblage total des jarres représente un nombre encore indéfini des différents types d'amphores Günsenin IV. Leur étude est donc susceptible de livrer des informations d'un intérêt majeur.

Ma proposition de classification des amphores byzantines a été adoptée universellement et que le nom Günsenin se trouve associé à la typologie des amphores $\left(10^{\mathrm{e}}-13^{\mathrm{e}}\right.$ siècles $)$. Dans ma thèse de doctorat, soutenue en $1990^{31}$, j'ai identifié trente-sept types d'amphores incluant des types intermédiaires pour les quatre premiers types. La découverte la plus importante associée avec le type Günsenin I est celle de son lieu de production, qui se trouve à Ganos (actuel Gaziköy) en Thrace. Les différences de forme des sous-types et l'étude des argiles suggèrent qu'il a pu y avoir d'autres lieux de production mais leur étendue semble circonscrite dans la région de la mer de Marmara. D'autre part, Yona Waksman en collaboration avec Skartsis, Kontogiannis, Todorova et Vaxevanis propose des lieux de production des types Günsenin I-III ${ }^{32}$; IIa et III. Quant aux amphores de Günsenin IV, leurs lieux de production sont toujours incertains, sauf des hypothèses raisonnables que l'on peut formuler.

J'apprécierais votre avis sur mes commentaires dans le second article dans ce volume, que j' ai entrepris de rédiger plus de trente ans après ma première communication sur ce sujet ${ }^{33}$.

\section{THE GÜNSENINN IV AND THEIR ARCHAEOLOGICAL CONTEXT (A.R.)}

An extended archaeological sounding was carried out in the northeastern corner of the platform where the elevation difference between the lower level and the platform is smaller than it is in other areas in order to assess the nature of the platform's fill, to reveal the architectural features, and to verify the stratigraphical sequence (Fig. 1).

33) Günsenin 2018, dans ce volume pp. 89-124. 


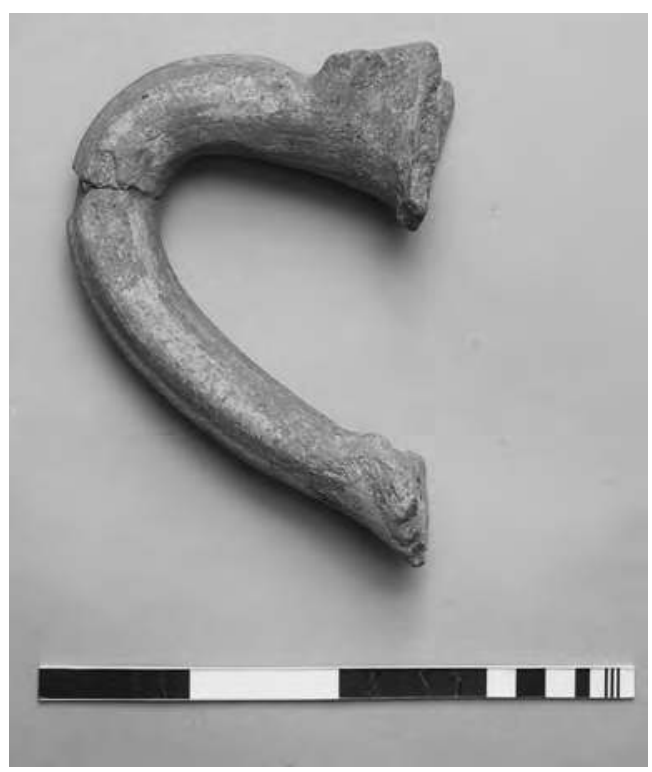

Fig. 12 : Anse d'amphore Günsenin IV, KY/16 ; US 2612, 2627

(Photo: Domenico Ventura. KYAP Archive).

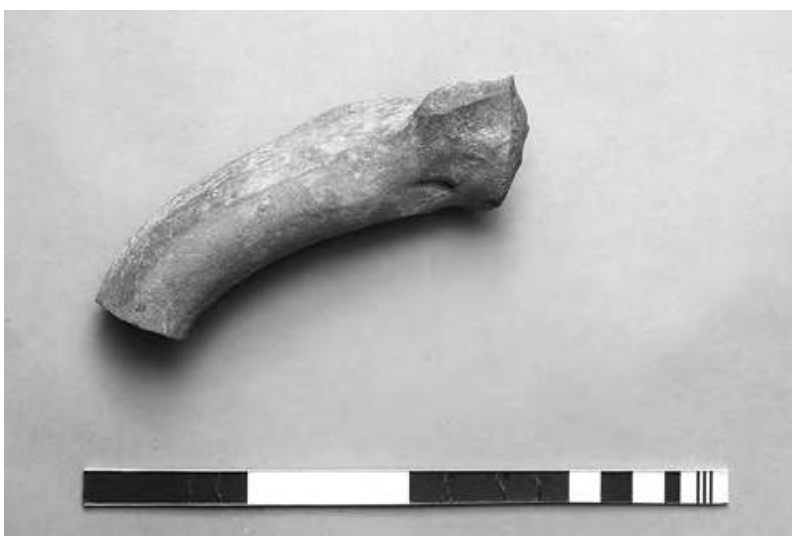

Fig. 14 : Anse d'amphore Günsenin IV, KY/16 ; US 2627 (Photo: Domenico Ventura. KYAP Archive).

Excavation of quadrants I3 and I2 in 2015 brought to light a rather well preserved phase identified as a late-Byzantine settlement area (Fig. 3a and 17). The eastern end of quadrant $I 2$ revealed the presence of a thick roof collapse. Here, a posthole reinforced with stones was excavated, and a set of intact ceramic objects including two candlesticks and an olive-brown glazed bowl were retrieved from around it and from beneath the roof collapse. They were found next to each other and it is likely they may have been used together. Their chronology

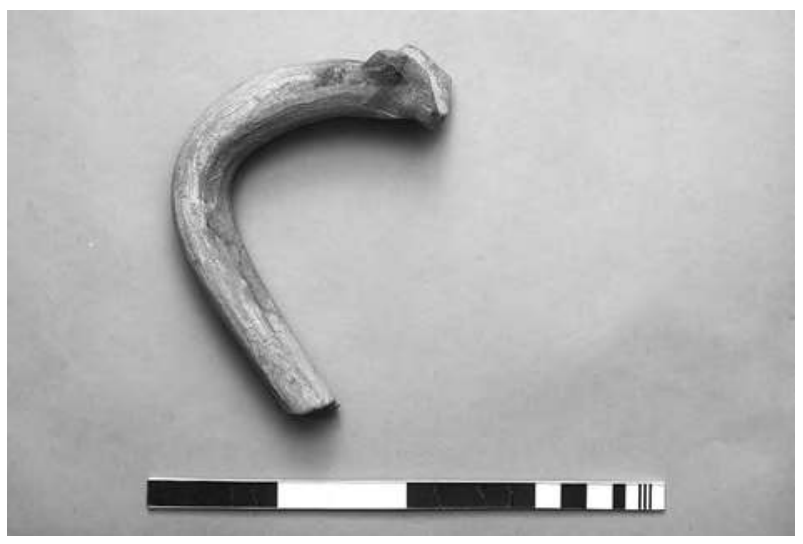

Fig. 13 : Anse d'amphore Günsenin IV, KY/16 ; US 2612 (Photo: Domenico Ventura. KYAP Archive).

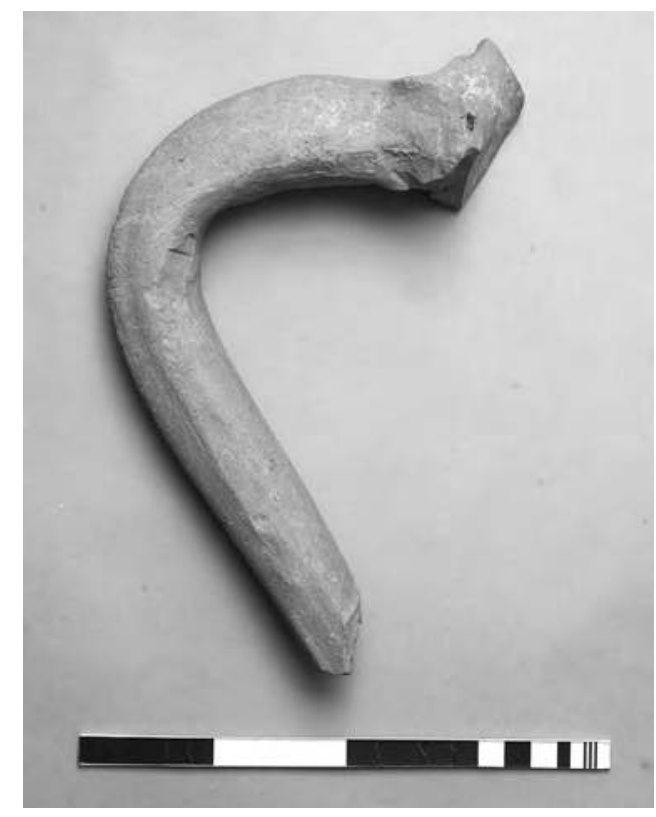

Fig. 15 : Anse d'amphore Günsenin IV, KY/16 ; US 2612 (Photo: Domenico Ventura. KYAP Archive).

ranges between the $8-9^{\text {th }}$ and $10^{\text {th }}$ centuries ${ }^{34}$. While it is difficult at this time to identify their function with certainty, the possibility that they served as part of a liturgical set should not be excluded.

The continuation of features to the west revealed the presence of a rather poorly executed porous calcareous floor with traces of simple construction (Fig. 1 and 17). An unusually large Günsenin IV amphora (KY/15; US 1504) was embedded into the floor (Fig. 3b). Not too far from the amphora, excavation of the layer underneath or by the end of the

34) R. Wohmann worked on the ceramics from this area. Ricci and Wohmann 2018 : 457, Fig. 3.22,23,24. 
calcareous floor, brought to light another feature that is unusual within known Byzantine-period Constantinopolitan archaeology. This was a garbage pit filled with large quantities of discarded material such as animal bones, shells, glass fragments, domestic pottery and tableware. Soil samples from the pit thus far showed a near-absence of archaeobotanical remains. Preliminary study of the material places its chronology to the $12^{\text {th }}$-early $14^{\text {th }}$ century ${ }^{35}$. Although excavation in this portion of the platform did not allow the exploration of the whole extent of the settlement area, which will hopefully be possible in the near future, some further considerations can be offered. The settlement area constituted by the calcareous floor level, the Günsenin IV amphora and the pit must have been covered in part by a simple roof, the traces of which are represented by the posthole. The roof, as well as the area as a whole, must have made use of the middle-Byzantine period platform walls and the portico above them, which defined the eastern side of the platform. The portico and the platform walls must not have been fully preserved by that time. On the basis of preliminary analysis of the archaeological finds, this area can be dated to the late $13^{\text {th }}$ century and into the early decades of the $14^{\text {th }}$ century.

Not too far from the settlement area of the late-Byzantine period stand the remains of the katholikon whose excavation begun in 2014 (Fig. 1) ${ }^{36}$. The building was equipped with rather prominent lateral entrances extending some $3 \mathrm{~m}$ beyond the external wall of the church. Excavation of the northern entrance showed a solid brick building technique with stepped foundations of mortar, ashlar blocks and brick. The foundations cut into the platform's earth fill. To the west and east of the entrance, the upper archaeological stratum (US 2612, 2627) was extremely rich in ceramics and amphora fragments in particular. Also, a number of amphora stoppers emerged from the excavation (Fig. 16). The same stratum by the northeastern wall of the church (quadrant H5) revealed an assemblage of archaeobotanical material consisting of wheat and linen seeds, a mill stone fragment, a small coarse ware stove, and a flint stone ${ }^{37}$. An intact elegant glazed sgraffito bowl found in the stratum can be dated between the second half of the $13^{\text {th }}$ and beginning of the $14^{\text {th }}$ centuries ${ }^{38}$. Excavation of the upper stratigraphical contexts around the northern

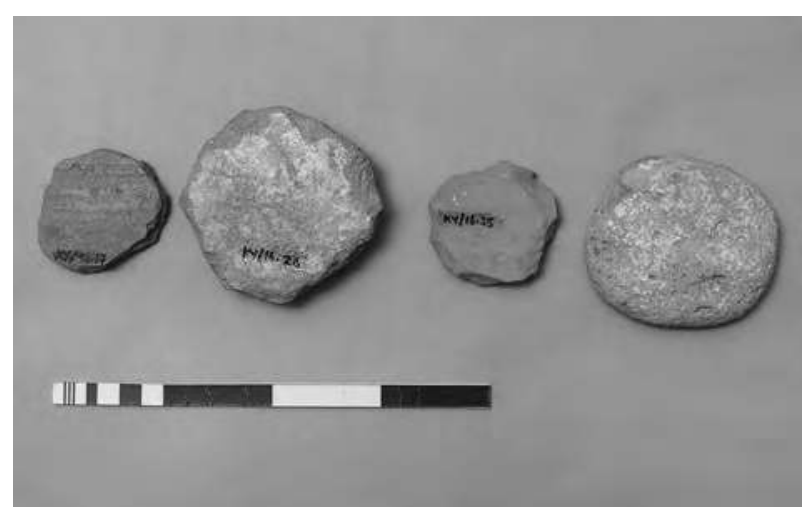

Fig. 16 : Les bouchons d'amphores (fragments d'amphores arrondies)

(Photo: Domenico Ventura. KYAP Archive).

entrance of the church may be consistent with productive activities, which, as in the case of the northeastern corner of the platform, indicate a late-Byzantine chronology. It will be important to continue excavations between the two areas in order to assess their connections.

The other Günsenin IV amphora (KY/10; US 1073) assembled in 2016 was retrieved in 2010 from a small-sized, rectangular plan chamber, added at a later time between the tower's northwestern and the platform's southwestern walls (Fig. 1). The chamber was built of poor masonry and was "hidden" against the middle-Byzantine monumental walls of the complex. The stratigraphical sequence and ceramic finds from the chamber have recently been published. Here too, the Günsenin IV along with a Glazed White Ware IV pitcher filled with wheat seeds have been interpreted to be in secondary use ${ }^{39}$.

The monastic establishment of Satyros at Küçükyalı is attested in the vita by David Niketas Paphlago, likely composed around the end of the $9^{\text {th }}$ century or in the early years of the $10^{\text {th }}$ century ${ }^{40}$. The monastery is mentioned again in the typikon for the monastery of Christ Pantokrator in Constantinople, dated to October 1136 . The inventory of proprieties assigned to the monastery by the emperor John II Komnenos reads: "The monastery of Satyros together with all conditional and unconditional rights belonging to them" ${ }^{41}$. Hence, the monastery and its community entered into the orbit of the Pantokrator from the early $12^{\text {th }}$ century onwards. It is unclear if and how the Pantokrator continued to

35) Ricci and Wohmann $2018: 457$.

36) Ricci, Forthcoming.

37) Preliminary report on the archaeobotanical finds, see Ulaş 2017 : 192-195.

38) I thank Rick Wohmann for his work on the piece.

39) Ricci and Wohmann $2018: 457$.

40) Simithies $2013:$ xi-xiii.

41) Jordan 2000, Vol. $2: 771$. 


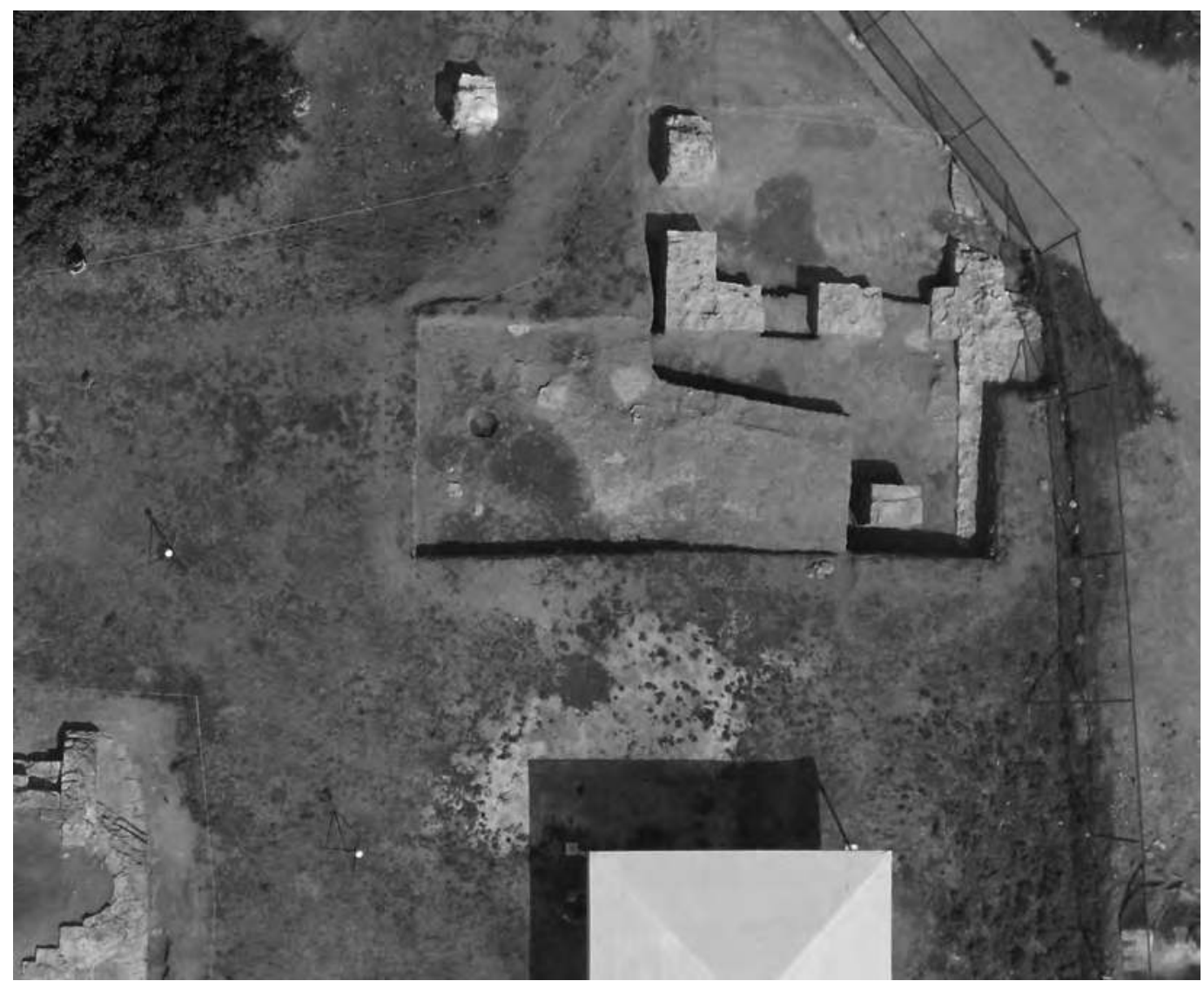

Fig. 17 : Vue zénithale du secteur I2 - I3 après la fouille en 2015 (KYAP Archive).

function as a monastic community during the Latin conquest of the city (1204-1261). However, Andronikos II Palaiologos (1282-1325) used the Pantokrator monastery also as an imperial burial site ${ }^{42}$. Further research on the sphere of influence and association between the Pantokrator monastery and its proprieties, the modes of production of proprieties as well as of goods exchanged in the Palaiologan period may contribute to a better understanding of the late-Byzantine phases at Satyros.

\section{CONCLUSION (?) (N.G.)}

Les différences de formes et de pâtes des trois amphores restituées et la récurrence de ce même phénomène sur des centaines d'anses et de fragments indiquent que les amphores trouvées à Küçükyalı appartiennent à des ateliers différents. On peut donc supposer que le monastère de Satyros avait développé des liens, notamment des relations commerciales avec plusieurs autres $\mathrm{m}$ onastères. Ce phénomène témoigne d'une activité importante au monastère au treizième siècle. Une autre constatation impor- tante est que les amphores ne portent pas de traces calcaires de la mer. On peut donc conclure qu'elles ne résultent pas d'un naufrage et qu'elles ont été volontairement transportées sur place. On peut enfin suggérer qu'il y avait un lien entre le monastère de Satyros et la cargaison de l'épave de Çamaltı Burnu I.

\section{En achevant ce travail préliminaire nous pou-} vons poser les questions suivantes :

- Comment les sous-types de Günsenin IV ont aussi voyagé jusque-là ?

- En partant de l'hypothèse que la cargaison de vin de l'épave de Çamaltı Burnu I appartenait à un monastère plusieurs questions peuvent être esquissées. Y avait-il un lien entre les deux sites ou bien avec les autres monastères ou, vu leur diversité de forme, avec les autres lieux de production des amphores? Par quelle voie est-ce que ces amphores ont débarqué au monastère de Satyros? Où était le port principal ?

42) Jordan 2000, Vol. $2: 726-727$. 
- La poursuite de la restauration des amphores, et un projet d'analyse de l'argile sont nécessaires pour identifier les lieux d'origine des jarres.

- La recherche est en cours !

\section{ADDENDUM (N.G.)}

A l'occasion de cet article, je voudrais bien attirer l'attention des lecteurs, en particulier ceux/celles qui s'occupent des fouilles d'archéologie urbaine de la période byzantine, sur le fait qu'on ne peut jamais prévoir d'avance tous les contextes où nos amphores peuvent être découvertes.

Dernièrement, je voudrais partager avec vous la contribution généreuse de van Doorninck :

"(...) Under one of the handles of the largest jar (KY/15; US 1504) are two long vertical lines cut into the clay seemingly before firing (Fig. 4c). You have at least a couple of examples of this among the larger jars from the Çamaltı Burnu I Wreck. This is another connection between the amphoras on your wreck and these jars..."

"You have an opportunity here to let our colleagues know a little about what we are learning from the Günsenin IV amphoras from the Çamaltı Burnu I Wreck about the size of the 3 amphoras of Küçükyalı: The approximate volume capacities of the three jars indicates what their weight capacities very probably were. The capacity of Byzantine amphoras was measured by the weight, not volume, of their contents. The basic unit of wine capacity was the mina, which equaled 3 Byzantine pounds. Capacity measurements of the table amphoras from the Çamaltı Burnu I Wreck have yielded a weight value for the mina of 915 grams at the time the jars were made. Ongoing measurements of the larger jars from the wreck indicate that they had weight capacities of from 20 to 100 minai at 10 minai intervals, as well as $33 \frac{1}{3}, 66^{2} / 3$ and $1331 / 3$ minai, the weight capacity of the largest of the jars. The largest of the Küçükyalı jars (KY/15; US 1504) very probably also had a weight capacity of $1331 / 3$ minai, which would be a body volume capacity of about 122 liters, and the other two jars (KY/10; US 1073, KY/16; US 2612, 2627) a weight capacity of 50 minai, which would be a body volume capacity of about 45.75 liters".

\section{REMERCIEMENTS (N.G. et A.R.)}

Je remercie Alessandra Ricci de m'avoir signalé les trouvailles d'amphores à Küçükyalı ainsi que Zeynep Kızıltan la directrice des Musées archéologiques d'Istanbul de m'avoir accordé les autorisations nécessaires pour leur étude et leur publication. Les contributions de Frederick H. van Doornick, Jr. étaient très précieuses.

Alessandra Ricci wishes to extend her thanks to Nergis Günsenin for her interest and contribution to the study of amphorae from Küçükyall. The visit by Frederick H. van Doorninck, Jr. at the site was illuminating for all of us. The Küçükyalı ArkeoPark team is grateful for the excavation permits (2014 through 2017) and thanks the Istanbul Archaeological Museum's Director, Sn. Zeynep S. Kızıltan. Taner Özgür worked on the conservation of the amphorae; Rick Wohmann assembled the GIV (KY/10; US1073) and worked on the ceramics from this and other areas; Nisan Lordoğlu excavated GIV (KY/15; US 1504); İlksen Baysaling and Domenico Ventura produced the photographs included in this article and Beril Sulamac1 with Görkem Günay worked on the drawings. Work and research at the Küçükyalı ArkeoPark was made possible by the ISTKA (Istanbul Development Agency); Koç University and The Koç University - Stavros Niarchos Foundation Center for Late Antique and Byzantine Studies and Dumbarton Oaks Center for Byzantine Studies (Trustees of Harvard University). Alessandra Ricci wishes also to thank the Alexander S. Onassis Foundation for the research fellowship (2016-2017). Finally, thanks are due to Aksel Tibet, editor of Anatolia Antiqua and to the anonymous reader.

N.G. et A.R. 


\section{BIBLIOGRAPHIE}

Adan-Bayewitz, D., 1986 : "The pottery from the Late Byzantine building (Stratum 4) and its implications", in. L. Levine et E. Netzer (éds), Excavations at Caesarea Maritima 1975, 1976, 1979. Final Report, Institute of Archaeology, Hebrew University of Jerusalem, Jerusalem : 91-100.

Bardill, J., 2004 : Brickstamps of Constantinople, Oxford Monographs on Classical Archaeology, Oxford, 2 Vol.

Eyice, S., 1959 : “İstanbul'da Abbâsi Saraylarının Benzeri Olarak Yapılan Bir Bizans Sarayı”, Belleten 23: 79-114.

Günsenin, N., 1990: Les amphores byzantines $\left(X^{e}-X I I I^{e}\right.$ siècles $)$ : typologie, production, circulation d'après les collections turques, Université Paris I (Panthéon-Sorbonne), Paris. Atelier national de reproduction des thèses de Lille III.

- 2001 : "L'épave de Çamaltı Burnu I (île de Marmara, Proconnèse) : résultats des années 1998-2000”, Anatolia Antiqua IX : 117-133.

- 2003 : "L'épave de Çamaltı Burnu I (île de Marmara, Proconnèse) : résultats des anneés 2001-2002", Anatolia Antiqua XI : 361-376.

- 2005: “A 13 ${ }^{\text {th }}$-Century Wine Carrier: Çamaltı Burnu, Turkey”, in G. Bass (éd.), Archaeology Beneath the Seven Seas, Thames and Hudson, England : 118-123.

- 2018: "La typologie des amphores Günsenin - Une mise au point nouvelle", dans ce volume pp. 89-124.

Günsenin (sous-presse) : "A Günsenin IV Amphora from Küçükyalı”, Bizantinistica. Rivista di studi bizantini e slavi.

Jordan, R., 2000 : "Pantokrator: Typikon of Emperor John II Komnenos for the Monastery of Christ Pantokrator in Constantinople", in J. Thomas et A. Constantinides Hero (éds.), Byzantine Monastic Foundation Documents, Dumbarton Oaks Studies XXXV, Washington D.C., Vols 1 et 5 . Vol 2 : 725-781.

Mamboury, E., 1920 : "Ruines Byzantines de Mara entre Maltépé et Bostandjik", Echos d'Orient 19 : 322-330.

Pargoire, V., 1901 : "Les monastères de Saint-Ignace et les cinq petit îlots de l'archipel des Princes", Bulletin de l'Institut archéologique russe de Constantinople 7 : 62-78.

Ricci, A., 1998 : "The Road from Baghdad to Constantinople and the case of the 'Bryas Palace', Istanbul', in L. Brubaker (éd.), Byzantium in the Ninth-century. Dead or Alive?, Society for the Promotion of Byzantine Studies 5, Ashgate : 131-149.

- 2012 : "Left behind: small sized objects from the Middle Byzantine complex of Satryos (Küçükyal1-Istanbul)", in B. Böhlendorf-Arslan et A. Ricci (éds.), BYZAS 15, Istanbul, Ege Yayınları : 147-161.

- 2017: "Infrastruttura, Produzione e Riutilizzo: il cantiere medio Bizantino a Küçükyalı (Istanbul) / Küçükyalı'da (Istanbul) Orta Bizans yerleşmesi: Üretim, altyapı ve yeniden kullanım", Arkeoloji ve Sanat / Journal of Archaeology and Art 154 : 135-146

Ricci (Forthcoming) : "Tracing the Capital's Asian Suburbs: Landscape, Architecture and Archaeology at the Küçükyalı ArkeoPark", Bizantinistica. Rivista di studi bizantini e slavi.

Ricci, A. et Y1lmaz A., 2016 : "Urban Archaeology and Community Engagement. The Küçükyalı ArkeoPark in Istanbul", in M. Alvarez, A. Yüksel et F. Go (éds.), Heritage Tourism Destinations: Preservation, Communication and Development, Oxon : 41-62.

Ricci, A. et Wohmann, R., 2018 : "Byzantine Contexts from the Asian Suburbs of Constantinople: Preliminary Remarks on the Ceramics and Archaeology at the Küçükyalı ArkeoPark (Istanbul)", in F. Yenişehirlioğlu (éd.), XI ${ }^{\text {th }}$ Congress AIECM3 on Medieval and Modern Period Mediterranean Ceramics Proceedings, Vols. I et II, 19-24 Octobre 2015, Antalya, Ankara, Vol I : 453-458.

Smithies, A., 2013 : Niketas David. The Life of Patriarch Ignatius, text and translation by Smithies A., with notes by Duffy, J., M., Dumbarton Oaks Text 13, Washington D.C.

Ulaş B., 2017 : "Küçükyalı: İstanbul'da bir Bizans Manastırının Tarımsal Ekonomisi", Arkeoloji ve Sanat I Journal of Archaeology and Art 154 : 192-195.

Volkov, I.V., 1992 : “O proishoždenii i evoljucii nekotoruch tipov srednevekovuch amfor", Donskie Drevnosti 1 : 143-157.

Waksman, S.Y., Skartsis, S.S., Kontogiannis, N.D., Todorova, E.P. et Vaxevanis, G., 2018 : "Investigating the origins of two main types of Middle and Late Byzantine amphorae", Journal of Archaeological Science : Reports, Vol. 21, Octobre 2018 : 1111-1121. 


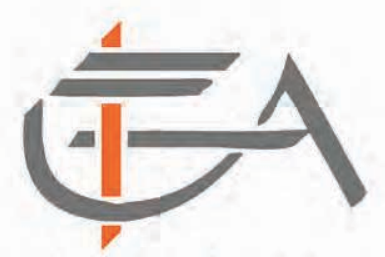

Institut Français d'Etudes Anatoliennes

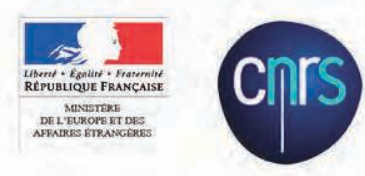

La citadelle de Tushpa (Van, Turquie).

ISBN: 978-2-36245-074-7

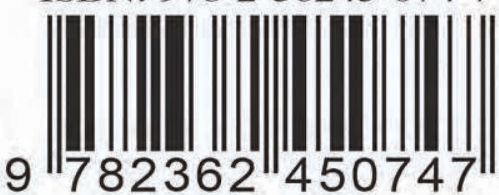

